\title{
FES Bike Race preparation to Cybathlon 2016 by EMA team: a short case report
}

\author{
Juliana Araujo Guimarães (1), Lucas Oliveira da Fonseca (1,2), Ana Carolina de Sousa \\ (2), Miguel Eduardo Gutierrez Paredes (2), George Andrew Brindeiro (2), Antônio Padilha \\ Lanari Bó (1,2), Emerson Fachin-Martins (1)
}

(1) NTAAI, Faculdade de Ceilândia, Universidade de Brasília, Brasília, Brazil; (2) LARA, Faculdade de Tecnologia, Universidade de Brasília, Brasília, Brazil

This article is distributed under the terms of the Creative Commons Attribution Noncommercial License (CC BY-NC 4.0) which permits any noncommercial use, distribution, and reproduction in any medium, provided the original author(s) and source are credited.

\begin{abstract}
FES-assisted cycling has been recommended to people struggling to emerge from a disability to more functioning life after spinal cord injury. Recommendations issued by a gowing number of scientific papershas promised toimprove body composition and physical activity levels, as well as to controlinvoluntary muscle response; favoring activity and participation which break new grounds in expanding locomotion, leisure and occupational options for people with paraplegia and tetraplegia. In this report we described our experience to select and prepare a pilot to compete in the FES Bike Race modality at Cybathlon 2016 in Kloten (Zurick). He was a man, 38 years old, with a complete spinal cord injury, level T9, three years of injury. He took part in a two preparation phases lasting respectively 18 and 12 weeks each: (1st) pre-FEScycling and a (2nd) FES-cycling. The 1st phase aimed to explore electrical stimulation response in the quadricps, hamstrings and gluteus muscles; searching for a standard muscular recruitment enable to propel the pedals of a trike. Following, in the 2 nd phase, stationary to mobile FES-cycling was performed at the same time the development of the automation and control systems were being incorporated in the trike. We adapted a commercial tadpole trycicle anda pilot controlled system. Although we had planned a three session by week protocol, for reasons of term and time to finish the trike development and be prepared to compete, in the last two weeks before the Cybatlhon an intense level of exercise was maintained. After the race, we noticedinflammatory signs on the left knee which later revealed a patella fracture. The video footage analysis confirmed ithappened during the race's first lap.
\end{abstract}

Key Words: electrical stimulation, spinal cord injury, FES cycling, risk evaluation, harms

Eur J Transl Myol 27 (4): 272-278

Correct methods to minimize the structural and functional body impairments primarily caused by spinal cord injury (SCI) are crucial to avoid secondary injury which could result in undesired outcomes for patients who survived the SCI and have to struggle emerging from the disability to a more functioning life. ${ }^{1}$ Nevertheless, the capacity obtained in a good acute care does not assure reaching the maximal performance if the patients are not engaged in a suitable rehabilitation program followed by attractive locomotion, leisure and occupational alternatives to continuously develop and maintain the residual capacities acquired after acute care. $^{2}$

Specially in the late period of SCI, after the first 2 years of injury, few attractive options are available to motivate people with SCI to remain engaged in a social structure, paving the way for activity-based therapies [assistive technologies such as Functional Electrical Stimulation (FES)-assisted cycling] to promote physical, mental and social recovery even in a chronic condition. ${ }^{3}$

Motivated by the increasing availability of scientific papers indicating benefits coming from FES-assisted cycling experiences and having been aware of the Cybathlon 2016 launch $^{4}$, we prepared a pilot to compete in the FES Bike Race modality.

\section{Methods \\ Recruitment process to potential pilots}

We conducted a public forum publicized among people with disabilities interested in coming out for sports. At this meeting, FES-assisted cycling was presented as an experimental activity-based therapy, which may be also considered as an assistive technology for locomotion, 


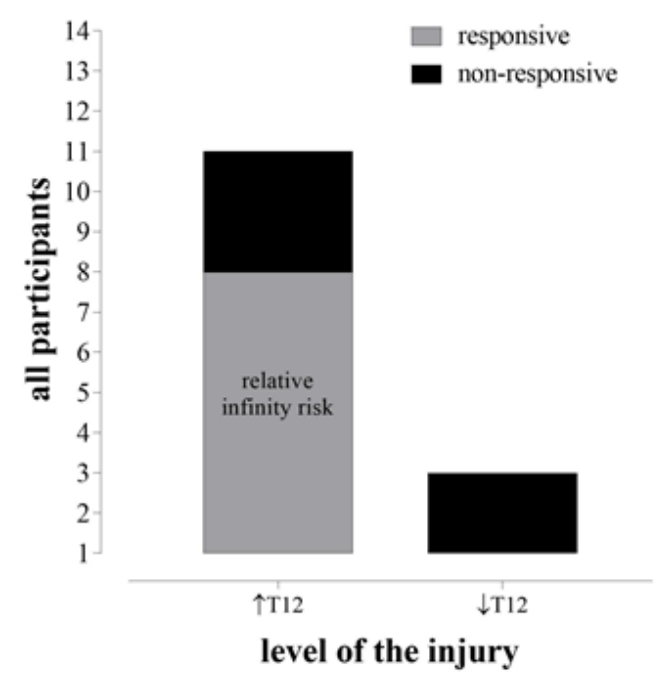

Fig 1. The frequency of distribution for participants responsive or non-responsive to the NMES by injury level. A significant $(p<0,05)$ association detected by Fisher's exact test suggests a relative infinity risk of the level of the injury predicting the responsiveness, choosing the method of Katz when the values are zero, adding 0.5 to all cells before calculating the relative risk and its confidence interval. Prism shows a floating note on the results page when it does this. In this case, it suggests us switch to the Koopman method.

leisure and sports. After explaining the technical principles of operation, and the benefits and risks evidenced by scientific articles published; we invited who had interest to take part in the experimental preparation, to apply for a screening test to identify the responsiveness to Neuromuscular Electrical Stimulation (NMES) using the parameters employed for FESassisted cycling. ${ }^{5}$

Fourteen subjects accepted to participate in the screening. All of them signed an informed consent (CAAE 50337215.1.0000.0030, approval number 1.413.934, local ethical committee).

\section{Screening test}

In the screening test, we had a sample aging from 29 to 41 years old, mostly composed by women (64\%), struggling with their paraplegies from 3 to 26 years of SCI, caused predominantly by trauma $(86 \%)$ in the levels above T12 (79\%) and with ASIA Impairment Scale (AIS) classified as A (complete, 64\%). The responsiveness to NMES was investigated by a biphasic rectangular pulse. The pulse width was set at $500 \mu$ s and the frequency at $50 \mathrm{~Hz}$, with intensity varing from 0 to $69 \mathrm{~mA}$ to trigger a muscle response different from $0 / 5$

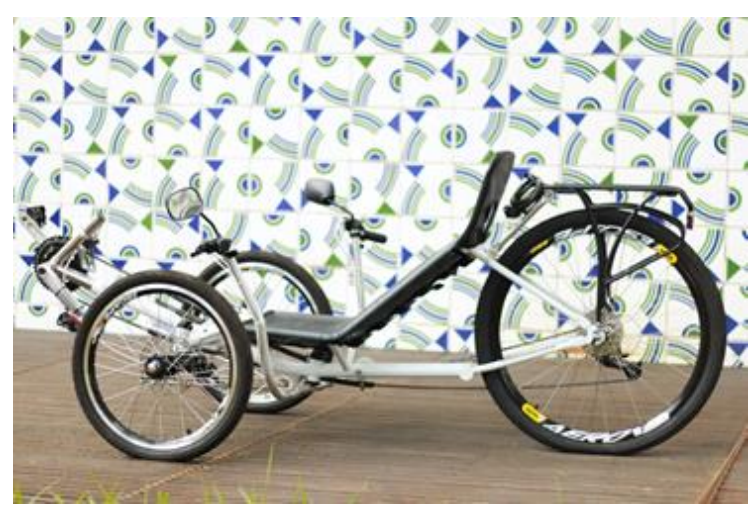

Fig 2. The tadpole trike adapted in this work for mobile FES cycling.

MRC (Medical Research Concil). At the end of the screening test, 8 participants $(57 \%)$ responded to the NMES. All of the responsive volunteers had traumatic injury above the T12 level. The Fisher's exact test detected a significant association between injury above the T12 level and responsiveness, accompanied by a relative infinity risk of the levels higher than T12 being a predictor of responsiveness (Figure 1). Body composition investigated by BMI (Body Mass Index) and atrophy in the lower limb muscles inferred by the right/left perimetry at thigh and calf levels did not associate with responsiveness ${ }^{6}$.

\section{Selection criteria}

Once identified, the responsive volunteers were invited to pursue the preparation to Cybathlon competition organized in two phases: (1st) pre-FES-cycling and (2nd) FES-cycling preparation. Only one of them who achieved all the steps in the two phases of the preparation would meet criteria to apply for the FES Bike Race on the Cybathlon Championship. Before the preparation, all the 8 candidates were assessed for skin wounds, muscular contractures, join stiffness and bone fragility by a medical doctor. From the responsive group, only two were considered elegible to take part in the preparation with progressive overloads because the others showed high risk for fractures during training (bone fragility detected by bone densitometry) and no tolerance to efforts. Bone densitometry was assessed by dual-energy x-ray absorptiometry (DXA). This technique results in 2-dimensional projections of the bones and BMD is calculated as mass per area (in $\mathrm{g} / \mathrm{cm} 2$ ). DXA measurements are commonly performed at the femoral neck and lumbar spine because the manufacturers provide softwares and reference values for these locations6. The criteria for the diagnosis of osteoporosis, low bone mass and normal bone mineral density followed those of the World Health Organization (WHO). The WHO reference for diagnosis of osteoporosis is a T-Score of -2.5 or lower in the proximal femur or lumbar spine. ${ }^{7}$ Between the two 


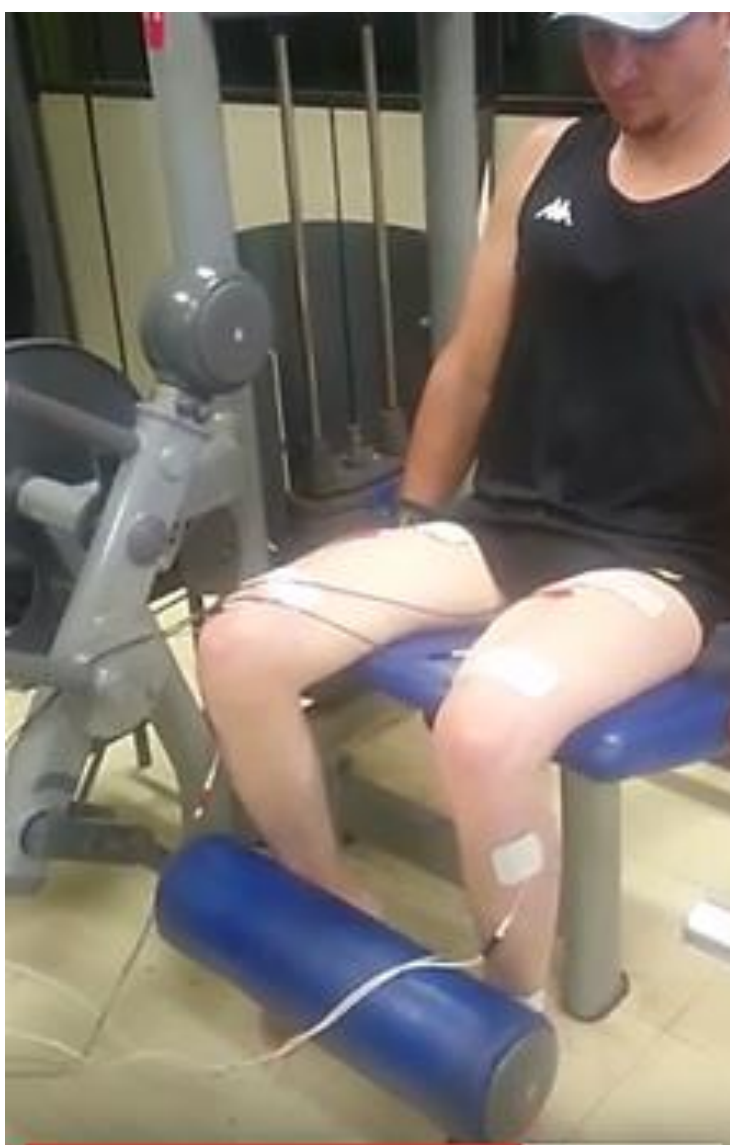

Fig 3. Photograph illustrating the inicial step of the 1st phase of progressive training in which the vastus lateralis muscle was activated together with the anterior tibialis muscle in isometric contractions during a sitting position.

elegible candidates, the one that developed the best muscular response to electrical stimulation without adverse effects as autonomic disrreflexia and better response during effort was selected as our pilot.

\section{Preparation of the selected pilot}

\section{The pilot}

Our pilot was a man of 38 years old, struggling with his paraplegy for 3 years of SCI, caused by trauma (gunshot) in the T9 level and with AIS classified as A (complete). He is $1.70 \mathrm{~m}$ and started the preparation weightening $65 \mathrm{Kg}$, resulting in a BMI of 22.49 (normal weight). He is currently a lawyer, but before the spinal cord injury he worked as a physical education teacher. Nowadays he is a high-performance athlete in adapted sailing and rowing. He was very excited and motivated to train FES-assisted cycling since the public forum and his engagement increased when he saw for the first time his paralyzed legs propelling the trike pedals. E.L. had no history of fragility fractures, no alteration in bone
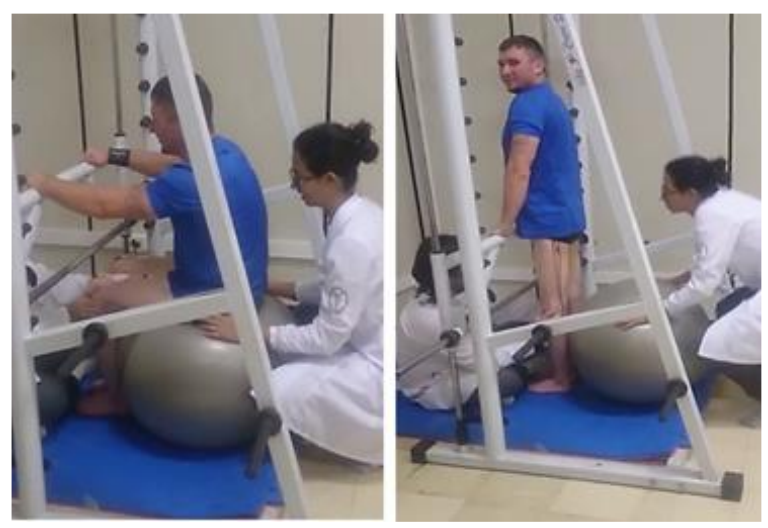

Fig 4. Photographs showing against gravity exercises by electrical stimulation of quadriceps, hamstrings and gluteus muscular groups during sit-to-stand transfer and standing maintenance position. The photograph recorded the beginning of the sitting position (left) and the end in the standing maintenance position (right).

mineral density (he presented values above the reference that determines bone fragility $\mathrm{T}$-score $>-2.5$ ), complete range of motion in lower limbs, no unhealed wounds, no autonomic dysreflexia and did not present excessive spasticity or adverse effects during effort and habituation period to electrical stimulation. He had no previous experience using electrical stimulation.

\section{Tricycle and control system}

A commercial recumbent trike (HP3 Trike, Brazil) with adjustable crankset position, $18 \mathrm{~kg}$ (approximately), $2 \mathrm{~m}$ (total length), 35 degrees seating position, and an 8speed regular gear system with chain tensioner was adapted for mobile FES cycling in this work (Figure 2).

The most relevant mechanical adaptation for FES cycling concerned the pedal. We designed and built an adapted pedal composed by supporting surfaces for the whole foot and a fixed vertical rod in order to secure the limb and prevent motion outside the sagittal plane. While using this device, the ankle joint is always kept at 90 degrees. The trike is instrumented with a wireless inertial sensor (3space, Yei Technology, USA) that enables estimation of crank position and angular speed. Both are sampled at $170 \mathrm{~Hz}$. A customized user interface enables the pilot to start and stop the system, as well as to modulate stimulation intensity. A stimulator (Rehastim, Hasomed, Germany), an embedded computer (Raspberry Pi 3, Raspberry Foundation, UK), and one battery complete the system. The software architecture is based on a multicore application that integrated all functionalities. Including all mechanical parts and hardware, the complete approximate cost of the system is USD 3,950. The control system is based on two layers: an inner loop 


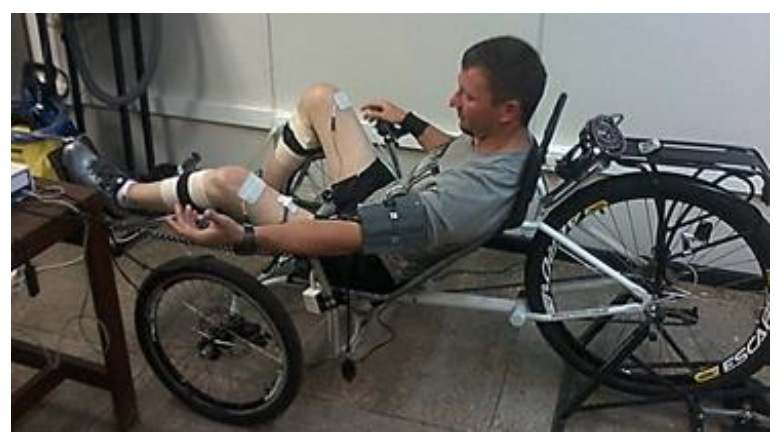

Fig 5. Photograph of stationary set up used during the initial training of the FES-cycling preparation.

controlling stimulation timing to produce cyclic leg movement and an outer loop that modulates stimulation intensity to control trike speed. The inner control loop is based on muscle-specific stimulation mapping as a function of crank angle position and velocity. For instance, extensor muscles are stimulated when leg extension is required. Furthermore, as described in Fonseca et al. (2016), ${ }^{8}$ the stimulation phases within the pedaling cycle are subject-specific. The outer loop is based on a PI cadence controller using pulse-width as the controllable variable.

\section{1st phase: pre-FES-cycling preparation}

In the beginning, our pilot was not able to perform the most common protocol recommended: 30 minutes of cycling at a cadence of $35 \mathrm{rpm}$. For this reason, the preFES-cycling preparation aimed to achieve a tolerance to electrical stimulation by means of electrically-induced knee extension training progressively. We planned a 18weeks preparation program, 3 times per week, 30 minutes each session using a four channel stimulator providing rectangular biphasic current (Dualpex 071 Quark, Brazil). As in the screening test, also during effective stimulation, frequency of $50 \mathrm{~Hz}$, intensity ranging from 0 to $69 \mathrm{~mA}$ and pulse width of $500 \mu \mathrm{s}$ were used without ramps. The intensity was increased from 0 up to a visual contraction (1/5). Electrodes (5 per $9 \mathrm{~cm} 2)$ were placed on the skin at a location in the quadriceps, hamstrings and glutei muscles where the best muscular response was found. The 1st phase started placing the pilot in a chair with fixed feet and performed a maximum 30 minutes of quadriceps isometric contractions each session (Figure 3 ) using a duty cycle ratio of 1:3. Then, for every cycle of 5 seconds a continuous contraction was evoked and, after 15 seconds of rest, the protocol of stimulation was repeated until fatigue or 30 minutes time out. When he became able to tolerate exercise for 30 minutes without fatigue, the duty cycle ratio was increased for 1:2. Finally, the last adaptation took place, where the ratio was set for 1:1. Fatigue was assessed by the visible loss of force and velocity during contractions. As the participant did
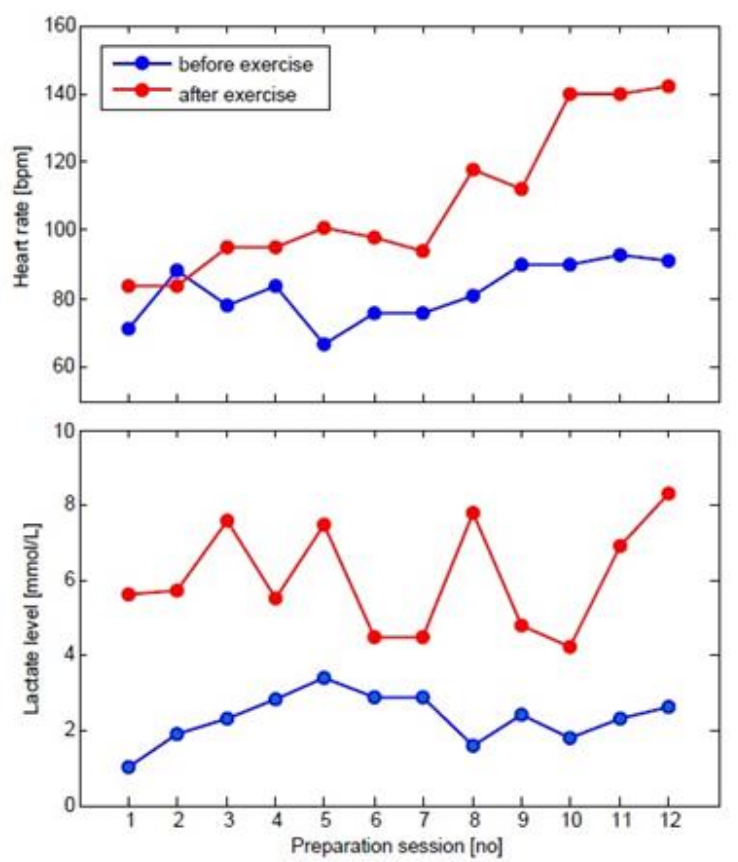

Fig 6. Heart rate and lactate levels before and after preparation sessions during the 12 weeks of FES-cycling preparation.

not realize pain or discomfort due to absence of sensation below the level of injury, he tolerated high intensities of electrical stimulation, achieving a maximum intensity of $95 \mathrm{~mA}$. Muscles strength was rated using the MRC scale ${ }^{7}$ of $0 / 5$ to $5 / 5$ in which $0 / 5$ represented no contraction; $1 / 5$ muscle flicker, but no movement; 2/5 possible movement, but not against gravity when the join was tested; $3 / 5$ possible movement against gravity, but not against resistance; $4 / 5$ possible movement against some resistance and 5/5 normal strength (movement against resistance). In the beginning of the protocol, he achieved an electrical stimulated contraction graduated as $2 / 5$, but it quickly led to fatigue, resulting in less than 10 minutes of quadriceps contraction. Following, during the 4th session, we achieved a $3 / 5$ electrical stimulated contraction of quadriceps, indicating possibility of a minimal support against gravity. So, we introduced electrical stimulation of other muscles (hamstrings and gluteous) by mean of dynamic contractions and functional tasks, such as sit-and-stand transfer and standing maintenance position as showed in fFigure 4 .

The main goal of the first weeks in the pre-FES-cycling preparation was achieving sufficient muscle strength to tolerate repeated electrical stimulated transfer and maintenance position during 30 minutes. In the transfer and maintenance position against gravity exercises, an 1:3 duty cycle ratio was performed, with 20 seconds of contraction composed by 4 seconds of ascending ramp (concentric contraction), 12 seconds of isometric 


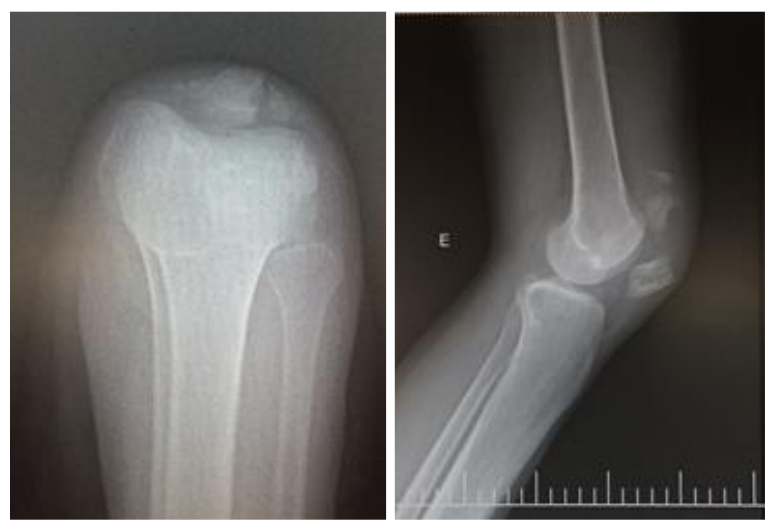

Fig 7. The $x$-ray images reaveling the cleft knee showing the comminuted fracture of the patella. The left image is a front view of the knee in 90 degree flexion, and the right image is a profile view in the sagital plane.

contraction, keeping the patient in the standing position; and 4 seconds of descending ramp (eccentric contraction) to the initial sitting position.

The described protocol was repeated 3 times per week with reccomendation of domiciliar electrical stimulation for all lower limb superficial muscles by the patient himself in the day off. When he was able to maintain 30 minutes without fatigue, he was then considered fit to begin the next phase preparation: FES cycling.

Despite achieving the goal at the 16th session (earlier in the 6th week), the planned 18-weeks program was performed until the end to give chance to the other 13 candidates to meet the requirements to the next phase. During this time, E.L. remained in the pre-FES-cycling preparation reproducing in full the achieved protocol for maintaining the accomplished performance.

\section{2nd phase: FES-cycling preparation}

The protocol was planned to be performed 3 times per week during 12 weeks ( 3 months before the Cybathlon 2016 competition). However, the engineers involved in the EMA team requested extra training sessions to complete the system development. In the last two weeks, our pilot was trained 5 times per week. Each training session lasted around one hour.

Surface electrical stimulation was conducted on the quadriceps (2 channels), hamstrings (1 channel) and glutei ( 1 channel) muscles. First stationary training was performed using resistance provided by a training roll to prevent free spin of wheel (Figure 5). On the second week, when the pilot was able to pedal during twenty minutes in this initial configuration, we introduced outdoor training with the current intensity controlled manually by the pilot. Closed-loop stimulation at a frequency of $20-30 \mathrm{~Hz}$, maximum pulse width of $300 \mu \mathrm{s}$, current intensity varying from $20 \mathrm{~mA}$ to $96 \mathrm{~mA}$ (minimum and maximum values) was applied. We monitored selected physiological variables during such training sessions, such as heart rate, arterial blood pressure, lactate level, and blood glucose level. The main challenge to provide a suitable monitoring of the exercise intensity with our pilot was his lack of perceived exertion and excessive determination towards the competition that created a favorable environment for overtraining, in particular, in the last two weeks before the FES Bike Race. We concluded that a moderate to intense level of exercise have been maintained over the last weeks. Nevertheless, during some preparation sessions high levels of training were reached, based on the estimation using lactate level (higher than 7 $\mathrm{mmol} / \mathrm{L}$ ) and heart rate (over $120 \mathrm{bpm}$ ) (Figure 6)

\section{Discussion}

\section{The Cybathlon 2016 by EMA team (Brazil)}

During the race, the parameters of stimulation were set to $30 \mathrm{~Hz}$ and $500 \mu \mathrm{s}$, with the intensity manually controlled by the pilot. He departed using values from 40 to $50 \mathrm{~mA}$ to glutei and quadriceps muscles, respectively, but in the end of the race the intensity reached values ranging from 86 to $96 \mathrm{~mA}$. Both hamstrings were not stimulated during the competition, since we observed an increase in strong spasms. However, later we concluded that the spasms were probably due to the overtraining.

In the 1st round, our pilot completed the first 25 meters in 13.1 seconds. During this round, a head camera captured the moment when our pilot suffered a patellar fracture (Figure 7). Despite this unfortunate event, he continued to pedal, since he had no knowledge regarding its occurrence. In addition, from the video records, we have observed that he was trying to compensate the FES system failure, helping manually his legs to push the pedal, a behavior that led judges to disqualify him, finishing his race.

As the fracture was not noticed at the 1th round, he participated in the 2 th final round when strong spasms were still occurring. Our rider achieved the eight overall position in the final classification of the competition.

The precise cause of knee fracture was not defined by the medical doctor. However his diagnosis suspicion was the overtraining (three to five times a week), once the pilot featured adequate bone mineral density. Several factors are thought to have contributed, such as high intensity of electrical stimulation in combination with strong muscle spasms which abruptly blocked the cycling movement, increased muscular strength induced by regular FES cycling in a closed kinematic chain with the knee in about 90 degrees of flexion (a biomechanical configuration that maximizes patellarfemoral contact), and other hypotheses. Indeed, we conclude that the risk of fracture as well as various precautions should be discussed and should be taken into consideration in future studies.

The indicated treatment was the surgical approach with fixation of the patella followed by physical therapy. The 
EMA team provided all the financial and operational support during the pilot's recovery period. The surgery took place on December 1st, 2016 and the rehabilitation period lasted until June 2017. Currently, the pilot has resumed the sports he practiced and the activities of daily living normally.

\section{FES cycling overtraining: risk evaluation and care}

Concerning the technological development, the proposed mechanical design presented intermediate geometry between seated (e.g. wheelchair-based FESassisted cycling) and fully recumbent positions. Our goal was to provide more comfort and visibility to the pilot. However, our pilot reported he was slipping on the seat, possibly affecting the performance and comfort. Transferring pilots to our system was also difficult and we are looking for solutions. Furthermore, the use of wireless sensors may reduce systems robustness due to lost packages, particularly when compared to wired systems. As an additional improvement, torque sensors may be used as a safety measure during spastic events. From a clinical point of view, and especially motivated by the harmful effect faced by a pilot who was certified by a medical doctor as featuring suitable health status to compete, we recommend caution with the enthusiasm and excitement coming from sports competition for a population in which extra efforts are hard to be monitored due the lack of sensorial recognition of the effort. Cooperation with other teams present at the first Cybathlon FES Cycling competition and beyond will provide further hints and tips. ${ }^{9-18}$ Our next step will be defining what are the thresholds levels of effort that are sufficiently high to provide structural and functional body adaptations to improve performance, while avoiding harmful overtraining.

\section{List of acronyms}

AIS - Abbreviated Injury Scale.

BMI - Body Mass Index

DXA - Dual Energy X-Ray Absorptiometry

FES - Functional Electrical Stimulation

MRC - Medical Research Council

NMES - Neuromuscular Electrical Stimulation

SCI - Spinal Cord Injuries

WHO - World Health Organization

\section{Author's contributions}

JAG was responsible for the pilot recruitment process, screening test and FES-cycling preparation of the selected pilot. She was also responsible by the healthcare support required along preparation, competition and post competition periods. LOdF designed, implemented and validated the control system, and was responsible for experimental setup implementation and technical data acquisition, as well as interpretation of obtained results. MEG was responsible for major mechanical adaptations on the trike and its parts, including the custom made pedal, and the experimental setup ACCdS implemented the control system and performed control validation tests. GB was responsible for software architecture design and implementation. APLB coordinated the entire project and the technological development, including both software, hardware, and mechanical aspects of the study, and contributed to the study design and interpretation of obtained results. EFM coordinated the financial support sponsored by CNPq (\#458671/20134), CAPES (Purpuse: 300665, \#88881.068134/2014-01) and FAPDF (\#193.000.639/2015), also contributed to the writing of the draft and did a critical revision and final approval of the article.

\section{Acknowledgments}

We thank professor U. Araujo who provided us an excellent work environment and a network of collaboration between CETEFE and the NTAAI. We also thank Charles Fattal and Christine Azevedo-Coste (INRIA by means of the CACAO associated team) by the knowledge exchange. Financial support sponsored by CNPq (\#458671/2013-4), CAPES (Purpuse: 300665, \#88881.068134/2014-01) and FAPDF (\#193.000. $639 / 2015)$.

\section{Conflict of Interest}

The authors declare no conflict of interests.

\section{Ethical Publication Statement}

We confirm that we have read the Journal's position on issues involved in ethical publication and affirm that this report is consistent with those guidelines.

\section{Corresponding Author}

Emerson Fachin-Martins, NTAAI, Faculdade de Ceilândia, Universidade de Brasília, Brasília, Brazil.

E-mail: emersonntaai@gmail.com.br

E-mails of co-authors

Juliana Araújo Guimarães: juguimaraesft@gmail.com Lucas Oliveira da Fonseca: lucasfonseca@lara.unb.br Ana Carolina Cardoso de Sousa: anacsousa@lara.unb.br Miguel Eduardo Gutierrez Paredes: meduag@lara.unb.br

George A. Brindeiro: georgebrindeiro@lara.unb.br Antônio Padilha Lanari Bó: antonio.plb@lara.unb.br

\section{References}

1. Sweis R, Biller J. Systemic Complications of Spinal Cord Injury. Curr Neurol Neurosci Rep 2017;17(2):1-8. doi:10.1007/s11910-017-0715-4.

2. Fu J, Wang H, Deng L, Li J. Exercise Training Promotes Functional Recovery after Spinal Cord Injury. Neural Plast 2016;2016:1-7. doi:10.1155/2016/4039580.

3. Yaşar E, Yılmaz B, Göktepe S, Kesikburun S. The effect of functional electrical stimulation cycling on late functional improvement in patients with 
chronic incomplete spinal cord injury. Spinal Cord 2015;53(12):866-869. doi:10.1038/sc.2015.19.

4. Cybathlon. Races \& rules. ETH Zurich. 2015;1. http://www.cybathlon.ethz.ch/content/dam/ethz/sp ecial-interest/conference-websites-dam/cybathlondam/documents/2014-10-03-cybathlon-rules.pdf.

5. Guimarães JA, Oliveira L, Fonseca D, et al. FESCycling in cases of paraplegia: a preliminary report Towards parameters and protocols to recommend FES-Cycling in cases of paraplegia: a preliminary report. Eur J Transl Myol. 2016;26:209-14. doi:10.4081/ejtm.2016.6085.

6. Eser P, Frotzler A, Zehnder Y, Denoth J. Fracture threshold in the femur and tibia of people with spinal cord injury as determined by peripheral quantitative computed tomography. Arch Phys Med Rehabil 2005;86(3):498-504. doi:10.1016/ j.apmr.2004.09.006.

7. Coupaud S, McLean, NA, Purcell $M$ et al. Decreases in bone mineral density at cortical and trabecular sites in the tibia and femur during the first year of spinal cord injury. Bone 2015;74:6975.

8. Fonseca L, Padilha A, Bo L, et al. Cadence tracking and disturbance rejection in FES cycling for paraplegic subjects: a case study. In: IFESS: International Functional Electrical Stimulation Society. La Grande Motte; 2016:lirmm 01412163.

9. Coste CA, Mayr W, Bijak M, et al. FES in Europe and Beyond: Current Translational Research. Eur J Transl Myol 2016;26(4):6369.

10. Peri E, Ambrosini E, Pedrocchi A, et al. Can FESAugmented Active Cycling Training Improve Locomotion in Post-Acute Elderly Stroke Patients? Eur J Transl Myol 2016;26:6063.

11. Azevedo Coste C, Bergeron V, Berkelman R, et al. Comparison of strategies and performance of functional electrical stimulation cycling in spinal cord injury pilots for competition in the first ever Cybathlon. Eur J Transl Myol 2017;27:251-4.

12. Berkelmans R, Woods B. Strategies and performances of Functional Electrical Stimulation Cycling using the BerkelBike with Spinal Cord Injury in a competition context (CYBATHLON). Eur J Transl Myol 2017;27:255-8.

13. Laubacher M, Aksöz EA, Bersch I, Hunt KJ. The road to Cybathlon 2016 - Functional electrical stimulation cycling Team IRPT/SPZ. Eur J Transl Myol 2017;27:259-64.

14. Sijobert B, Fattal C, Daubigney A, AzevedoCoste B. Participation to the first Cybathlon: an overview of the FREEWHEELS team FES-cycling solution. Eur J Transl Myol 2017;27:265-71.

15. Metani A, Popovic-Maneski L, Mateo S, et al. Functional electrical stimulation cycling strategies tested during preparation for the First Cybathlon Competition - a practical report from team ENS de Lyon. Eur J Transl Myol 2017;27:279-88.

16. McDaniel J, Lombardo LM, Foglyano KM, et al. Cycle Training Using Implanted Neural Prostheses: Team Cleveland. Eur J Transl Myol 2017;27:289-94.

17. Arnin J, Yamsa-ard T, Triponyuwasin $\mathrm{P}$, Wongsawat Y. Development of practical functional electrical stimulation cycling systems based on an electromyography study of the Cybathlon 2016. Eur J Transl Myol 2017;27:295301.

18. Leung WC, Tong RKY, Wang X, et al. The Effectiveness of Functional Electrical Stimulation (FES) in On-Off Mode for enhancing the cycling performance of Team Phoenix at 2016 Cybathlon. Eur J Transl Myo 2017;27:302-6. 